\title{
Definability as Hypercomputational Effect
}

\author{
S. Barry Cooper ${ }^{1}$ \\ School of Mathematics, University of Leeds \\ Leeds LS2 9JT, U.K.
}

\begin{abstract}
The classical simulation of physical processes using standard models of computation is fraught with problems. On the other hand, attempts at modelling real-world computation with the aim of isolating its hypercomputational content have struggled to convince. We argue that a better basic understanding can be achieved through computability theoretic deconstruction of those physical phenomena most resistant to classical simulation. From this we may be able to better assess whether the hypercomputational enterprise is proleptic computer science, or of mainly philosophical interest.
\end{abstract}

Key words: computability, definability, hypercomputation

PACS: 01.70.+w, 01.75.+m, 02.10.Ab

\section{Introduction}

In the face of new challenges to familiar computational paradigms, the role of classical computability theory, based on Turing machines and the ChurchTuring Thesis, has appeared to be a largely reactive one. Reflective of the growing body of literature openly questioning the relevance of classical models of computation is the following quotation from Goldin and Wegner's [1] paper from $\mathrm{CiE} 2005$ :

1 The author wishes to thank István Németi for a number of helpful comments on a first draft of this paper, which have led to a number of improvements.

Preparation of this article partially supported by EPSRC Research Grant $\mathrm{EP} / \mathrm{C} 001389 / 1$ Extensions of Embeddings in the Local Turing Universe, and by NSFC Grand International Joint Project Grant No. 60310213 New Directions in Theory and Applications of Models of Computation. 
"It is time to recognise that today's computing applications, such as web services, intelligent agents, operating systems, and graphical user interfaces, cannot be modeled by Turing machines; alternative models are needed."

This echoes and extends van Leeuwen and Wiedermann's 2] observation 2 that "the classical Turing paradigm may no longer be fully appropriate to capture all features of present-day computing."

In contrast to this, one has Martin Davis [3] confidently asserting that:

"The great success of modern computers as all-purpose algorithm-executing engines embodying Turing's universal computer in physical form, makes it extremely plausible that the abstract theory of computability gives the correct answer to the question 'What is a computation?', and, by itself, makes the existence of any more general form of computation extremely doubtful."

This has become a hard position to maintain. If only, as Philip Welch [4] points out, because logical proofs of the impossibility of hypercomputation "may be akin to proofs that fairies are logically impossible: damn hard to be convincing."

In 1998 Jack Copeland [25] claimed to have rediscovered in Turing's 1939 paper [7], based on his 1938 thesis at Princeton, a previously unsuspected hypercomputational agenda, based on the familiar oracle Turing machine - an exegesis which seemed to throw the embattled classical computability theorist a lifeline. But Davis [3] was having none of it:

"It is perfectly plain in the context of Turing's dissertation, that Omachines were introduced simply to solve a specific technical problem about definability of sets of natural numbers. There is not the faintest hint that Turing was making a proposal about a machine to be built. ... It makes no sense to imagine that he was thinking about actual machines to compute the uncomputable."

But just say we do have misgivings about universality of the universal Turing machine - surely it makes sense to look more closely at problematic computational situations, using all the technical sophistication which computability theory puts at our disposal. May that not lead to computational models with sufficient explanatory power to throw light on current controversies? Whatever our interpretation of Turing's real views on mechanical

$\overline{2}$ See also the related paper by the same authors on Relativistic computers and non-uniform complexity theory. In: Calude et al (eds.) UMC 2002. Lecture Notes in Computer Science Vol. 2509, Springer-Verlag, Berlin, 2002. pp. 287-299. 
computability (and in [8] I have indicated that these were not as simple as sometimes thought), Copeland has effectively spotlighted the potential for mathematically analysing the computationally complex in terms of its algorithmic content. As we argued in [9]:

"If one abstracts from the Universe its information content, structured via the basic ... fundamental laws of nature, one obtains a particular ... manifestation of the Turing universe ..., within which vague questions attain a precise analogue of quite plausible validity."

What is needed is for the same level of deconstructive analysis Turing applied to the intuitive concept of computation to be applied to hypercomputation.

This article is motivated by two important questions. One is the computabilitytheoretic question concerning the nature of the relationship between computation and (hyper)computational effects. The other is the more down-to-earth question of whether hypercomputation, if it actually occurs, can be successfully harnessed to human computational needs. This is how Martin Davis [10] puts it in relation to Etesi and Nemeti's discussion [11] of relativistic behaviour near black holes:

"Of course, even assuming that all this really does correspond to the actual universe in which we live, there is still the question of whether an actual device to take advantage of this phenomenon is possible." 3

Or, more negatively (Davis [3]):

"In any case, a useable physical representation of an uncomputable function, would require a revolutionary new physical theory, and one that it would be impossible to verify because of the inherent limitations of physical measurement."

Is Davis right:4 Of course, in principle, he might well be. What may change that is a better understanding of the computational character of physical processes. In which case, the answer to the second question will give us a better idea of whether the hypercomputational enterprise is proleptic computer science, or philosophy.

The approach of Németi and Dávid [12] is a rather different one from that taken here. Theirs is based on what they describe as 'a major paradigm shift in our physical world-view as well as our cosmological one'. They seek to give scientific substance to the 'fairies' Welch refers to: an enterprise which, as was

3 Davis adding "But the theoretical question is certainly of interest."

4 Davis' particular doubts here are addressed on a number of levels in the István Németi and Gyula Dávid article [12] in this volume. 
argued in [5], is 'damn hard to be convincing', but which we would expect to provide an important element in the establishment of a larger paradigm shift.

\section{Neural mapping and real-world hypercomputation}

If one is looking for a context which is both widely suspected of transcending the standard Turing model, and of whose inner workings we have a high level of detailed knowledge, we need look no further than the human brain. Turing himself [13] talks about 'the inadequacy of 'reason' unsupported by common sense", and in his 1939 paper [7] says:

"Mathematical reasoning may be regarded ... as the exercise of a combination of ...intuition and ingenuity. ... In pre-Gödel times it was thought by some that all the intuitive judgements of mathematics could be replaced by a finite number of ... rules. The necessity for intuition would then be entirely eliminated. In our discussions, however, we have gone to the opposite extreme and eliminated not intuition but ingenuity"

Teuscher [14] identifies the gap to be filled by suitably extended models:

"People have started thinking about the possibility that simulating the mind in silicon might be impossible — or at least impossible using today's methods. Should we first forget about computers and look closer at the gray stuff in the brain, since the actual knowledge of the brain is severely fragmented and many details are still not at all understood?"

Part of the brain's potential for enrichment of our modelling of the computationally complex lies in the way it seems to successfully deal with the sort of imaging of the real world we would dearly like our computing machines to perform. More important, the brain shows the capacity to perform representations of mental imaging to enable recursive development of complex conceptual structures. At the same time, new techniques for relating structural and functional features of the brain, for example, using positron emission scan (PET) or a functional magnetic resonance imaging scan (fMRI), bring us much closer to obtaining useful models.

Connectionist models of computation based on the workings of the human brain have come a long way since Turing's [15] discussion of 'unorganised machines' (see Jack Copeland and Diane Proudfoot's article [16] On Alan Turing's Anticipation of Connectionism), and McCulloch and Pitts' seminal paper [17] on neural nets. But despite the growth of computational neuroscience as an active research area, putting together ingredients from both artificial neural networks and neurophysiology, something does seem to be missing. This 
leads Rodney Brooks [18] to allude to the fact that "neither AI nor Alife has produced artifacts that could be confused with a living organism for more than an instant." Again: "... neural networks alone cannot do the job" opines Steven Pinker, going on to describe [19, p.124] "a kind of mental fecundity called recursion":

"We humans can take an entire proposition and give it a role in some larger proposition. Then we can take the larger proposition and embed it in a still-larger one. Not only did the baby eat the slug, but the father saw the baby eat the slug, and I wonder whether the father saw the baby eat the slug, the father knows that I wonder whether he saw the baby eat the slug, and I can guess that the father knows that I wonder whether he saw the baby eat the slug, and so on."

We are good at devising computational models capable of imaging, and of going some way to emulate how the brain comes up with neural patterns representing quite complex formations. But the mechanisms the brain uses to represent such patterns and relate them in complex ways is more elusive. What makes the sort of recursion Stephen Pinker has in mind so difficult to get to grips with at the structural level, is that it seems wound up with the puzzle of consciousness and its relationship to emotions and feelings. Antonio Damasio [20, p.169] has a nice description of the hierarchical development of a particular instance of consciousness within the brain (or, rather, 'organism'), interacting with some external object:

"... both organism and object are mapped as neural patterns, in firstorder maps; all of these neural patterns can become images. ... The sensorimotor maps pertaining to the object cause changes in the maps pertaining to the organism. ... [These] changes ... can be re-represented in yet other maps (second-order maps) which thus represent the relationship of object and organism. ... The neural patterns transiently formed in second-order maps can become mental images, no less so than the neural patterns in first-order maps."

Notice that what is envisaged is the re-representation of neural patterns formed across some region of the brain, in such a way that they can have a computational relevance in forming new patterns. This is where the clear demarcation between computation and computational effect becomes blurred. The key conception is of computational loops incorporating these 'second-order' aspects of the computation itself. Building on this one can derive a plausible schematic picture of of the global workings of the brain.

Considering how complex a structure the human brain is, it is surprising one does not find more features needing reflecting in any basic computational model based on it. However, a thorough trawl through the literature, and 
one's own experiences, fails to bring to light anything that might be held up as computational principle transcending in a fundamental way what we have already identified. The key ingredients we expect in a model are imaging, parallelism, interconnectivity, and a counterpart to the second-order recursions pointed to above.

Mathematically, the imaging appears to be dependent on the parallelism and interconnectivity. This is what connectionist models are strong on. The recursions are not so easy to model, though. Looked at logically, one has representations of complex patternings of neural events underlying which there is no clear local mechanism, but for which one would expect a description in terms of the structures pertaining. Looked at physically, such descriptions appear to emerge, and be associated with (but not exclusively) the sort of non-linear mathematics governing the emergence of new relations from chaotic environments. This leads us to turn the picture of re-representations of mental imaging as a describable mapping on its head, and think (see [21]) in terms of descriptions in terms of a structure defining, and hence determining, the mental re-representations.

Looking at this more closely, what seems to be happening is that the brain stores away not just the image, but a route to accessing that image as a whole. This is what people who specialise in memorising very long numbers seem to display - rather than attempting to go directly into the detailed memory of a given number, they use simple representational tricks to call the entire number up. Here is how Damasio summarises the process (and the quotation from [20, p.170] is worth giving in full):

"As the brain forms images of an object — such as a face, a melody, a toothache, the memory of an event — and as the images of the object affect the state of the organism, yet another level of brain structure creates a swift nonverbal account of the events that are taking place in the varied brain regions activated as a consequence of the objectorganism interaction. The mapping of the object-related consequences occurs in first-order neural maps representing the proto-self and object; the account of the causal relationship between object and organism can only be captured in second-order neural maps. ... one might say that the swift, second-order nonverbal account narrates a story: that of the organism caught in the act of representing its own changing state as it goes about representing something else."

We think we know what is happening now? One needs only attempt a translation of our 'understanding' into an engineering context to realise we still have some way to go.

Other hypercomputational models are even more resistant to deconstruction. 
Roughly speaking, the more plausible the proposed instance of hypercomputational context, the more clearly present are all the ingredients we have drawn out above, and the less amenable it is to deconstructive inspection. For instance, we have Etesi and Nemeti's closely argued paper [22], describing how relativistic considerations (involving the actuality of such things as large rotating black holes in galactic nuclei) may lead to effectively computable functions which are not Turing computable. The argument even puts up a credible resistance to Martin Davis' destructive agenda in [10], but finding out exactly what is hidden behind the complexities of the nonlinear mathematics involved is a hard task 5 This tends to be true, of course, of all the real-world, physically based examples, starting with the suggestion of Georg Kreisel [23, p.143], that a collision problem related to the 3 -body problem might give "an analog computation of a non-recursive function (by repeating collision experiments sufficiently often)."

\section{Parallelism and interconnectivity}

So the building of routes through the Turing barrier appear blocked by parallel barriers to deconstruction of familiar processes. It is like exploration without maps and other navigational aids. Adventurous investigators may on occasion happen on strange and unrecognised computational territory, but when they return, their 'discoveries' do not fit with the familiar scientific landscape, and there is no consensus about how to interpret their accounts. This certainly applies to how we view the role of parallelism and interconnectivity within computational processes.

As early as 1988 Paul Smolensky observed in his influential Behavioral and Brain Sciences paper [24, p.3] that:

"There is a reasonable chance that connectionist models will lead to the development of new somewhat-general-purpose self-programming, massively parallel analog computers, and a new theory of analog parallel computation: they may possibly even challenge the strong construal of Church's Thesis as the claim that the class of well-defined computations is exhausted by those of Turing machines."

The classical computability theorist will be wary of elevating what may be to the applied scientist very important operational considerations into something more fundamental. One has to be very careful about claiming that some example of computational parallelism cannot be simulated by a Turing machine.

5 Although the new paper of Németi and Dávid in the present volume seeks to explain more thoroughly how and why such general relativistic computers work. 
As is well-known (see, for example, David Deutsch [30, p.210]), the massive parallelism delivered by quantum computation as it is currently abstracted is perfectly well contained within the classical sequential model. What happens when one raises the level of internal connectivity? It is still not clear that without analogue elements, such as in real world physical processes, you cannot come up with adequate descriptions of the algorithmic content of a given connectionist model of computation. Maybe external interaction will leave the classical model floundering? This idea runs through a number of hypercomputational proposals, including Copeland's [25] rediscovery of oracle Turing machines. But as Davis so effectively did, all our classical computability theorist has to do is to widen the definition of "internal" — that is to make the modelling more inclusive - to shepherd the proposed new paradigm back into the classical fold.

There are limits to this though, determined by those on the success of hypercomputational modelling.

Goldin and Wegner [27] certainly have the modelling of more than internal connectivity in mind when they quote from Robin Milner's 1991 Turing Award lecture [28, p.80]:

"Through the seventies, I became convinced that a theory of concurrency and interaction requires a new conceptual framework, not just a refinement of what we find natural for sequential computing."

In [1], for instance, Goldin and Wegner are not just talking about parallelism. And the inherent vagueness of examples they quote both stretch the mathematical analysis, and the reductionist agenda which feeds on that, to its limits:

"One example of a problem that is not algorithmic is the following instruction from a recipe [29]: 'toss lightly until the mixture is crumbly.' This problem is not algorithmic because it is impossible for a computer to know how long to mix: this may depend on conditions such as humidity that cannot be predicted with certainty ahead of time. In the function-based mathematical worldview, all inputs must be specified at the start of the computation, preventing the kind of feedback that would be necessary to determine when it's time to stop mixing."

Our intention here is not to rehash the familiar over-worked arguments over whether hypercomputation exists or not. In section 11 we settled on reasons to bypass those. I just want to give us reasons to look more closely at how the real world expresses those aspects of hypercomputational mental activity which we isolated in section 2, and for which a better understanding may well be necessary for the next computing revolution. 


\section{Hypercomputation computationally simulated}

It is when we look more closely at the operational benefits of connectionist models that we get an inkling of what is new in a more basic sense.

Viewing schematically, we do not have to look further than Turing machines to observe computably generated objects which are not themselves computable. Shine a bright light on the graph of a computable function over the natural numbers, and its shadow is likely to be incomputable - or more precisely, the projection of a suitable computable binary relation over the numbers produces an incomputable set of numbers. So it seems we already knew that you get incomputable objects by selectively observing algorithmic processes. The only problem with our Turing machine example is one seems to need infinitely much time, which makes the computable simulation of incomputability interesting, but difficult to connect with our own world.

However, if we put a little flesh on the algorithmic skeleton that is the Turing machine, we can get something which looks of more immediate interest. We can view fractals, and other mathematical objects generated according to simple algorithmic rules as computer simulations. The extraordinary richness of structure we observe is matched by the as yet unsolved problems of showing that aspects of, for instance, the Mandelbrot and certain Julia sets are computable (see [31, and, for contrast, 32], 33], 34]). Of course, such mathematical examples provide a metaphor for the way real-world complexity is generated by the iteration of simple algorithmic content, and nicely illustrate how the analysis of apparently finitary objects rests on uncompleted infinities and the mathematics of the infinite.

There is a fruitful two-way dialectic based on this interplay between computation and natural phenomenon. Nature is systematically raided nowadays for computational metaphors. Implicit in this is an assumption that nature can do something for us not yet delivered by unaided computational models. This 'something' may be of a purely operational character, or may well rest on the sort of simulation of hypercomputation talked about above. Whatever it is, it is enough to fuel the huge current interest in areas such as artificial neural networks, cellular automata (derived from self-reproduction), membrane computing (based on biological cells), evolutionary computing, L-systems (multicellular organisms), swarm computing, molecular computing, and so on. One does not even need to extract the metaphor to computationally exploit this 'something' - one can ask the natural world itself to do part of the computation. Of course, the analogue approach tends to niche applications, and is unlikely to ever deliver a relacement for our desktop computer! In general, a lot of ingenuity needs to go into making natural phenomena compute more than themselves. 
Both the computational metaphors, and the direct exploitation of natural phenomena, seem to suffer from a basic deficiency, for different reasons. The full complexity of human problem solving depends on the breaking up of problems into parts which can be attacked with varying techniques, a sort of 'divide and conquer' approach, involving loops in reasoning and interactions between conceptual frameworks. This lack of modularity and subroutines seems to be a feature of natural computation as we know it, particularly clearly in the case of quantum and relativistic computation, or analogue computing. It is not that these features are absent from the real world. Far from it. But because we do not fully understand how they arise, we cannot replicate them in our models, nor ensure stability in direct exploitation of their physical embodiments.

An important footnote to what we have said above is that one does not have to limit usable simulations to those classically modelled via enumerations of data. And if we think in terms of mental visualisations, this is just as well. As Turing pointed out (see Hodges [35, p.361]) in a talk to the London Mathematical Society, in February 20, 1947:

"... if a machine is expected to be infallible, it cannot also be intelligent.

There are several theorems which say almost exactly that."

It is reasonable to think in terms of appropriate levels of adjustment and approximation for particular simulations, all capturable in classical computability theoretic hierarchies. This does not just mean that factual mistakes may need correcting, but refers to the whole process of detailed imaging of objects external and internal to the organism.

\section{Definability and hypercomputational effects observed}

The picture we have built up seems to be a paradoxical one. On the one hand we believe that the classical dichotomy between computable and computably enumerable sets is reflected in the real world in the chasm between phenomena we can compute and those we can simulate. We believe that a simulation does hypercomputationally that a Turing machine does not - produces observable infinity in finite time. But for this to have a real impact on our conception of what happens in reality, we need valid model 6 that actually compute relative to the non-local heightened information content. To be precise, we have models which elevate information content, and models which exploit this, but no overall cohesive picture. And it seems that the problem we had understanding the mechanics of consciousness and the local representation of

${ }_{6}$ By which we mean abstract, mathematical models, which do not appeal to undeconstructed elements of the reality they are intended to model. 
non-local neural phenomena in the brain has a precise parallel in the difficulty we have knitting together these two types of computational model. If you come up with a model which elevates information content (such as in Beggs and Tucker [36]), you will probably be told by someone that it is unrealisable. If you admit a rich pre-existent information content in your model to give scope to the interactivity we identified earlier (as do most of Davis' targets in [3]), the sceptic will have enough to deride its hypercomputational content as also being pre-existent. Let us give our paradox a name:

Davis' Paradox: Hypercomputation is dependant on interactivity within a domain of rich information content. But the existence of rich information content depends on hypercomputation.

Returning to our section 2 neural avatar of hypercomputation, notice that we find it hard to locate intelligence as something that resides purely within the autonomous brain. The richness of mental activity appears as an extension of an equally complex world with which it interacts. Here is Brooks [37] again, drawing out this inclusiveness in a particulary dramatic way:

"Real computational systems are not rational agents that take inputs, compute logically, and produce outputs ... It is hard to draw the line at what is intelligence and what is environmental interaction. In a sense, it does not really matter which is which, as all intelligent systems must be situated in some world or other if they are to be useful entities."

In fact, Brooks [38, p.139] plausibly argues that there is a realistic approach to AI involving no internally generated representations, but rather using "the world as its own model".

Let us now find a way past Davis' paradox, in a way that validates the intuitions of both Martin Davis and Jack Copeland. And gives us a mathematical model which translates the dichotomy we started this section with into one between nature captured by linear mathematics, and emergent phenomena (see [21]). The idea is to provide a universe of scientific information content within which it makes sense to situate algorithmic content sufficient to model that extractable from the material world. If you like, we want to provide a playing field upon which the scientifically observed game of existence can be played out. This kind of model will be an extension of the classical model correctly favoured by Davis, and with the same kind of potential inclusiveness, but more in tune with what we actually observe. And this model, rather than being over-stretched by Goldin and Wegner's examples of real-world interactivity referred to in section 3 , will positively lap them up.

Our model has as its underlying domain the set of all real numbers, within which the scientist commonly describes the material universe. The algorithmic 
content the scientist is intent on extracting is inclusively modelled in terms of Turing's extension of his machine model of computation to one capturing computation relative to auxiliary information, accessed piecemeal via real numbers, and outputting real numbers again. The real numbers involved are dealt with via finitary approximations, of course. One can find the details of this extended Turing model — what is often called the Turing universe, familiar to us via its associated Turing degree structure - in most current computability theory texts (such as [39], [40], or [41]).

The intention now is to use this mathematical model, in conjunction with our scientific understanding of the workings of the neural setting, to, on the one hand, give precision to interpretations of what is happening in the brain, and on the other, to reinforce our perceptions of the relevance of the Turing model, and to refine those of the model's inevitable limitations. There are three obvious qualifications to be noted in claiming relevance for the extended Turing model. The first and most fundamental qualification is that the model can only help us understand the underlying information content of a given computationally complex environment, and that one must look more carefully to see what this means in terms of constraints on the detailed workings of the organism or physical context in question. The second is that the information content present in a given physical example will at most present some relatively small substructure of the full mathematical model. And perhaps most problematically, there will always be concern about what part of the algorithmic content of the Turing universe is relevant. Considerations of time, space and observed algorithmic content may entail refinements of the model, and even involve movement to different but related models which maintain the overall analytical framework, but lead to rather different detailed conclusions. But let us get back to our intended dialectic between mathematical and physical.

Firstly, note how all our predictively implementable understanding of the world is via its algorithmic content. From a post-Church-Turing perspective, this is almost tautological. And most of what we scientifically understand in a wider sense is built on that algorithmic content. Our higher order descriptions of reality tend to be shaped within this predictive framework. It may be that the weather, say, is not highly predictable in detail, but we do believe that climatic intricacies are emergent from a context whose local ingredients have well-understood algorithmic content. This is not to say there are not scientific puzzles, which have still to be brought within this dominant overall picture. Viewing the sub-atomic world from above, we only see the emergence in the form constrained randomness, while the underlying local algorithmic content and its connections to what we observe cannot be easily grasped. But the neural case is more accessible (which is partly why we chose it), and offers a more immediately productive environment in which to work out parallels between mathematical explanation and apprehensions of physical processes. 
What is most interesting about the Turing universe are questions about exactly which global relations are definable in terms of its local structure given by the locally applied algorithmic content, or Turing reductions - using natural language familiar to us from its precise formulations in first-order logid 7 . What is particularly puzzling about the human mind is how it manipulates mental images, formed non-locally within the brain, into higher-order conceptual formations. The use of language, peculiar to our own species, seems to be connected to this ability, but not a prerequisite for it. Now, on the one hand, the fact that language can be used to capture such higher-order formations points to a connection with language and definability. On the other, the well established fact that language is not actually necessary for this, makes us look more closely at what a description or definition actually provides in the way of structured understanding. The background to this will be our earlier discussion [21] of the link between emergence and definability, within which we seek to situate our discussion of the gap in our understanding of neural representation.

The claim is that a description gives us a handle on a complex emergent relation, in that the form of the description provides a recipe for the simulation of the phenomenon described. The brain may store a number of images in the form of non-local simulations. It may also store descriptions of how to access those images. Those descriptions must be in some sense centrally located, and involve mental images of how to algorithmically access mental features which may be far from algorithmic in their origins and current simulative character. In doing this, the brain does not necessarily use language. What is involved is yet another layer of cognitive function, with what we can think of as a recipe given by the description imaged, and becoming part of what Damasio calls core consciousness. More graphically: What is happening is that the recipe embodied in the definition of the emergent relation which is the location of the mental image, and which provides the means to reconstructing the relation and its link to the core, makes the relation part of a framework within which it can be recalled and become part of a hierarchical interactive network of concepts, ideas, facts, and other stored mental images. What is striking is that when we examine how we ourselves construct and implement such recipes, we notice that the related image has a 'feeling'. It is this which seems to identify, and to be the subjective apprehension of, its entry into a core neural structure, and provides the means to what Damasio identifies as our 'ownership' over the emergent relation via its description. Here is how Damasio [20, p.148] puts it:

"The perspective for a melody you hear or for an object you touch is,

7 The underlying concept of definability, basic to this article, is that familiar to logicians, where a relation on a structure is termed definable if it can be described in terms of the atomic relations of the structure using formal language, usually first-order, based on that of everyday usage. 
quite naturally, the perspective of your organism because it is drawn on the modifications that your organism undergoes during the events of hearing or touching. As for the sense of ownership of images and the sense of agency over those images, they, too, are a direct consequence of the machinations which create perspective. They are inherent in those machinations as foundational sensory evidence. Later, our creative and educated brains eventually clarify the evidence in the form of subsequent inferences, which also become known to us."

But where does this structure, which is accompanied by such symptomatic feelings, come from? And how does it relate to our mathematical model? Within the mind and its wider context of the physical universe, the core consciousness which is the key to this structuring is itself immanently emergent, while at the same time being the key to new activities. In a sense, the core, Damasio's 'core consciousness', is itself defined by its own activities and the storing of descriptions of its relationship to neural formations ([20, p.183]):

"Beyond providing a feeling of knowing and enhancement of the object, the images of knowing, assisted by memory and reasoning, form the basis for simple nonverbal inferences which strengthen the process of core consciousness. These inferences reveal, for instance, the close linkage between the regulation of life and the processing of images which is implicit in the sense of individual perspective. Ownership is hidden, as it were, within the sense of perspective, ready to be made clear when the following inference can be made: if these images have the perspective of this body I now feel, then these images are in my body - they are mine."

What the extended Turing model provides is the deconstruction of specific mental processes into what may be characterised as auxiliary (internally or externally originating) hypercomputational input, algorithmic content (in terms of locally specific neural connections), and the recording and utilisation of relevant descriptions representing definitions in terms of the more global neural environment of mental inter-relationships.

\section{Conclusions}

The closest anyone has got so far to actual computers with recognisably hypercomputational ingredients is by surfing physical reality in some way. There is a widespread suspicion that the world cannot be satisfactorily located within the standard computational model. This is not necessary for one to have an interest in new computational paradigms - as in the case of quantum computation, there may be very important operational benefits, even though there 
is an underlying classical model. But this suspicion gets stronger the more difficult it is to divorce ones computational approach from its real-world origins.

One way forward is to utilise the physical world's rich potential for computation, without worrying too much about understanding the underlying rules of the game. The likely success of this approach may be limited - it takes ingenuity 8 to get a natural process to compute more than itself - but may be the best we can do in the short to medium term. Or ever!

Let us take an analogy. The domestication of horses around five or six thousand years ago brought a revolution in transportation, only achieved through a creative interaction between humans and the natural world. At that time, trying to understand the principles underlying the equine organism in order to synthesise an artificial horse was unthinkable. But a few thousand years later there was enough understanding of scientific basics to underpin the invention of the "iron horse", leading, amongst other things, to the opening up of many previously isolated parts of the world to people with no riding skills whatsoever.

The moral of this seems to be that perhaps there is a great deal to be got from an ad hoc computational relationship with the real world. But that we should not be daunted by the sheer wonder natural structures inspire in us. It may be that the human brain, as an emergent phenomenon, has an intimate relationship with processes which are not easily simulable over significantly shorter time-scales than those to which natural evolution is subject - a kind of earthy victory of the functionalist over the computationalist view of the human mind. Maybe we will never build an artificial brain, anymore than we can make an artificial horse. But this does not mean we may not one day have a good enough understanding of basic hypercomputational principles to build computers - or firstly non-classical mathematical models of computation which do things undreamt of today.

Rodney Brooks [38, p.139] gets to have the last word:

"I, and others, believe that human level intelligence is too complex and little understood to be correctly decomposed into the right subpieces at the moment and that even if we knew the subpieces we still wouldn't know the right interfaces between them. Furthermore, we will never understand how to decompose human level intelligence until we've had a lot of practice with simpler level intelligences."

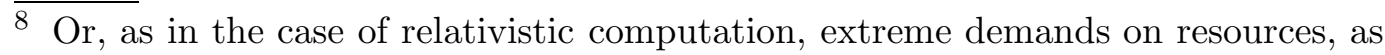
surmised by Németi and Dávid [12]. 


\section{References}

[1] Goldin D. and Wegner P. (2005) The Church-Turing Thesis: Breaking the Myth. In CiE 2005: New Computational Paradigms: Papers presented at the conference in Amsterdam, June 8-12, 2005 (Cooper S. B., Löwe B., Torenvliet L., eds.), Lecture Notes in Computer Science No. 3526, Springer-Verlag.

[2] van Leeuwen J. and Wiedermann J. (2000) The Turing Machine Paradigm in Contemporary Computing. In Mathematics Unlimited - 2001 and Beyond (Enquist B. and Schmidt W., eds.), Lecture Notes in Computer Science, Springer-Verlag.

[3] Davis M. (2004) The myth of hypercomputation. In Teuscher C. (ed.) Alan Turing: Life and legacy of a great thinker. Springer-Verlag, Berlin, Heidelberg.

[4] Welch P. (2004) On the possibility, or otherwise, of hypercomputation. British J. for Philosophy of Science, 55, 739-746.

[5] Cooper S. B. and Odifreddi P. (2003) Incomputability in Nature. In Computability and Models: Perspectives East and West (S. B. Cooper and S. S. Goncharov, eds.), Kluwer Academic/ Plenum Publishers, New York, Boston, Dordrecht, London, Moscow, pp. 137-160.

[6] Copeland J. (1998) Turing's O-machines, Penrose, Searle, and the brain. Analysis, 58, 128-38.

[7] Turing A. M. (1939) Systems of logic based on ordinals. Proc. London Math. Soc. (2) 45, pp. 161-228. Reprinted in A. M. Turing, Collected Works: Mathematical Logic (Gandy R. O. and Yates C. E. M., eds.), North-Holland, Amsterdam, New York, Oxford, Tokyo, 2001 pp. 81-148.

[8] Cooper, S. B. (2005) The incomputable Alan Turing. In Turing 2004: A celebration of his life and achievements, electronically published by the British Computer Society.

[9] Cooper, S. B. (1998) Beyond Gödel's Theorem: Turing nonrigidity revisited. In Logic Colloquium '95 (eds. Makowsky, J. A. and Ravve, E. V.), Proceedings of the Annual European Summer Meeting of the Association of Symbolic Logic, Haifa, Israel, August 9-18, 1995, Lecture Notes in Logic, vol. 11, Springer, Berlin, New York, Tokyo, pp. 44-50.

[10] Davis M. (to appear) Computability, computation and the real world.

[11] Etesi G. and Németi I. (2002) Non-Turing computability via Malament-Hogarth space-times. Int. J. Theoretical Physics 41, 341-370.

[12] Németi I. and Dávid G. (2005) Relativistic computers and the Turing barrier. This volume.

[13] Turing A. M. (1954) Solvable and Unsolvable Problems. Penguin Science News 31, 7-23. 
[14] Teuscher C. (2004) Turing's connectionism. In Alan Turing: Life and legacy of a great thinker (Teuscher C. ed.) . Springer-Verlag, Berlin, Heidelberg, pp. 499-529.

[15] Turing A. M. (1948) Intelligent machinery. National Physical Laboratory Report. In Machine Intelligence 5 (Meltzer B. and Michie D., eds.), Edinburgh University Press, Edinburgh, 1969, pp. 3-23. Reprinted in A. M. Turing, Collected Works: Mechanical Intelligence (Ince D. C., ed.), North-Holland, Amsterdam, New York, Oxford, Tokyo, 1992.

[16] Copeland J. and Proudfoot D. (1996) On Alan Turing's anticipation of connectionism. Synthese, 108, 361-377. Reprinted in Artificial Intelligence: Critical Concepts in Cognitive Science (R. Chrisley, ed), Volume 2: Symbolic AI. Routledge, London, 2000.

[17] McCulloch W. and Pitts W. (2003) A logical calculus of the ideas immanent in nervous activity. Bull. Math. Biophys. 5, 115-133.

[18] Brooks R. (2001) The relationship between matter and life. Nature 409, 409411.

[19] Pinker S. (1997) How the Mind Works. W. W. Norton, New York.

[20] Damasio A. (1999) The Feeling Of What Happens. Harcourt, Orlando, FL.

[21] Cooper S. B. (2005) Computability and emergence. In Mathematical Problems from Applied Logic. New Logics for the XXI-st Century II (eds. Gabbay D., Goncharov S., Zakharyaschev M.) Kluwer/Springer International Mathematical Series, Vol. 5 .

[22] Etesi G. and Németi I. (2002) Non-Turing computations via Malament-Hogarth space-times. Int. J. Theoretical Phys. 41, 341-370.

[23] Kreisel G. (1970) Church's Thesis: a kind of reducibility axiom for constructive mathematics. In: Intuitionism and Proof Theory: Proceedings of the Summer Conference at Buffalo N.Y. 1968 (Kino A., Myhill J., and Vesley R. E., eds.), North-Holland, Amsterdam, pp. 121-150.

[24] Smolensky P. (1988) On the proper treatment of connectionism. Behavioral and Brain Sciences 11, 1-74.

[25] Copeland J. (1998) Turing's O-machines, Penrose, Searle, and the brain. Analysis, 58, 128-38.

[26] Copeland J. (2000) Narrow versus wide mechanism: Including a re-examination of Turing's views on the mind-machine issue. J. of Phil. 96, 5-32.

[27] Goldin D. and Wegner P. (2003) Computation Beyond Turing Machines: seeking appropriate methods to model computing and human thought. Communications of the ACM 46, 100-102.

[28] Milner R. (1993) Elements of interaction:Turing award lecture. Communications of the ACM 36, 78-89. 
[29] Knuth D. (1968) The Art of Computer Programming, Vol. 1: Fundamental Algorithms. Addison-Wesley.

[30] Deutsch D. (1997) The Fabric of Reality. Penguin, London, New York.

[31] Hertling P. (2005) Is the Mandelbrot set computable? Math. Logic Quarterly $\mathbf{5 1}, 5-18$.

[32] Rettinger R. and Weihrauch K. (2003) The computational complexity of some Julia sets. In Proceedings of the 35th Annual ACM Symposium on Theory of Computing, San Diego, California, USA, June 9-11, 2003 (Goemans M. X., ed.), ACM Press, New York, pp.177-185.

[33] Braverman M. (2005) Hyperbolic Julia Sets are Poly-Time Computable. In Proceedings of the 6th Workshop on Computability and Complexity in Analysis, Wittenberg, Germany, August 16-20, 2004 (Brattka V., Staiger L. and Weihrauch K., eds.), ENTCS v.120, Elsevier, Amsterdam, pp.17-30.

[34] Rettinger R. (2005) A fast algorithm for Julia sets of hyperbolic rational functions. In Proceedings of the 6th Workshop on Computability and Complexity in Analysis, Wittenberg, Germany, August 16-20, 2004 (Brattka V., Staiger L. and Weihrauch K., eds.), ENTCS v.120, Elsevier, Amsterdam, pp.145-157.

[35] Hodges A. (1992) Alan Turing: The Enigma. Vintage, London, Melbourne, Johannesburg.

[36] Beggs E. J. and Tucker J. V. (2004) Computations via experiments with kinematic systems. Technical Report 5-2004, Department of Computer Science, University of Wales Swansea, March 2004.

[37] Brooks R. A. (1991) Intelligence Without Reason. A.I. Memo No. 1293, The A.I. Laboratory, M.I.T., Cambridge, MA, April 1991.

[38] Brooks R. A. (1987) Intelligence Without Representations. Artificial Intelligence, 47, 139-159.

[39] Cooper S. B. (2004) Computability Theory. Chapman \& Hall/ CRC Press, Boca Raton, FL, New York, London.

[40] Odifreddi P. (1989) Classical Recursion Theory. North-Holland, Amsterdam, New York, Oxford.

[41] Soare R. I. (1987) Recursively Enumerable Sets and Degrees. Springer-Verlag, Heidelberg. 